Faculty of Mathematical Sciences

\section{University of Twente}

University for Technical and Social Sciences
P.O. Box 217

7500 AE Enschede

The Netherlands

Phone: +31-53-4893400

Fax: $+31-53-4893114$

Email: memo@math.utwente.nl

Memorandum No. 1523

Order picking in carousel systems

under the nearest item heuristic

N. Litvak, ${ }^{1}$ I. Adan, ${ }^{2}$ J. Wessels ${ }^{3}$ ANd W.H.M. Zijm

APRIL 2000

ISSN 0169-2690 


\title{
Order Picking in Carousel Systems under the Nearest Item Heuristic
}

\author{
N. Litvak, I. Adan, J. Wessels and W.H.M. Zijm
}

March 30, 2000

\begin{abstract}
A carousel is a computer controlled warehousing system, which is widely used to store small and medium sized goods. One of the most important performance characteristics of such systems is the pick time of an order, which mostly depends on the travel time of the carousel. In this paper we consider some reasonable heuristics for order picking. In particular we establish properties of the Nearest Item (NI) heuristic. This one is frequently used in practice. We derive tight upper bounds for the travel time under the NI heuristic, and closed form expressions for its mean and variance. We also present a simple two-moment approximation for the distribution of the travel time. In addition, we find the mean, variance and distribution for the number of turns.
\end{abstract}

\section{Introduction}

A carousel is an automated warehousing system consisting of a large number of shelves or drawers rotating in a closed loop in either direction. Such systems are used for storage and retrieval of small and medium sized goods. The picker has a fixed position in front of the carousel, which rotates the required items to the picker. The advantage of such systems is that the picker has time for sorting, packing, labeling etc., while the carousel is rotating.

One of the most important performance characteristics of carousel systems is the total time needed to pick an order. Orders are represented by a list of items. The list specifies the type and retrieval quantity of each item. Ideally, the items should be picked in a sequence minimizing the total pick time, which is the travel time plus the pure pick time. The latter obviously does not depend on the pick strategy. Hence, we only have to consider the travel time in order to minimize the total pick time.

Bartoldi and Platzman [1] and Stern [6] study the optimal pick strategy for a carousel system. They show that there are only $2 n$ candidate sequences, where $n$ is the number of positions to be retrieved. It implies that an optimal route can always be found in linear time. Extensions to the algorithms of Bartholdi and Platzman and Stern have been presented by Van den Berg [2], who also reviews recent literature on carousel systems, 
as part of a general overview on planning and control algorithms for warehousing systems. Rouwenhorst et al. [5] provide some stochastic upper bounds for the optimal route. Their upper bounds are proved to be rather tight. Nevertheless, neither the probability distribution nor tight upper bounds for the minimum travel time have been obtained yet.

In their paper Bartoldi and Platzman [1] also consider some simple heuristics for a carousel system. One of these heuristics is the Nearest Item (NI) heuristic, where the next item to be picked is always the nearest one. In particular, the authors prove that the travel time under the NI heuristic is never greater than one rotation of the carousel.

In the present paper we also study the NI heuristic. We improve the upper bound of Bartoldi and Platzman [1] for the travel time and we show that the new upper bound is tight. Using probabilistic arguments we obtain a formula for the mean travel time and the distribution of the number of turns under the assumption of uniformly distributed pick positions. We also study the remaining travel time and the remaining number of turns after picking some items, i.e., when there is a known empty space at one side of the picker's position. A recursive procedure is developed to obtain closed-form expressions for the mean and variance of the travel time and the number of turns conditioned on the size of the empty space at one side of the picker's position. We further approximate the distribution of the travel time under the NI heuristic by a beta-distribution with the same support, mean and variance. This approximation is validated by simulation and it appears to be quite accurate.

The paper is organized as follows. In the next section we introduce the model and some notation. In Section 3 we study upper bounds for the travel time under the NI heuristic. In particular, we improve an upper bound of Bartoldi and Platzman [1]. In Section 4 we obtain a formula for the mean travel time using probabilistic arguments. In Section 5 we develop a recursive procedure to derive a closed-form expression for the mean travel time conditioned on the size of the empty space at one side of the picker's position. In Section 6 we use this procedure to find the variance for the travel time. In Section 7 we present a two-moment approximation for the travel time under the NI heuristic. Further in Section 8 we find the distribution for the number of turns under the NI heuristic, and in Section 10 we recursively find the conditional distribution for the number of turns. In the final section we briefly discuss our results.

\section{Carousel model}

Following Bartoldi and Platzman [1] and Rouwenhorst et al. [5] we represent a carousel as a circle of length 1 . Let the random variable $U_{0}$ be the picker's starting point and the random variable $U_{i}$, where $i=1,2, \ldots, n$, be the position of the $i$ th item. We suppose that the $U_{i}$ 's, $i=1,2, \ldots, n$, are independent and uniformly distributed on $[0,1)$. In the sequel we will denote by

$$
\omega=\left(\omega_{0}, \omega_{1}, \ldots, \omega_{n}\right) \in[0,1)^{n+1}
$$

a realization of the random vector $\left(U_{0}, U_{1}, \ldots, U_{n}\right)$. 


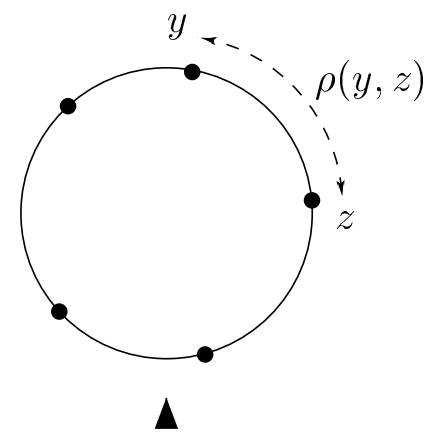

Figure 1: A carousel system.

The presentation will become more clear, when we act as if the picker travels to the pick positions instead of the other way around.

We denote the shortest distance between the positions $y$ and $z$ on a carousel by $\rho(y, z)$ (see Fig. 1). We assume that the acceleration time of the carousel is negligible or that it is assigned to the pick time. Hence, the travel distance can be identified with the travel time.

This completes the model description. In the next section we will explore the travel time under the NI heuristic.

\section{Upper bounds for the travel time}

The main object in this section is to establish an upper bound for the travel time under the NI heuristic and to prove its tightness. The NI heuristic can be described as follows (cf. Bartoldi and Platzman [1]): Always rotate to the nearest item to be retrieved. An important feature of the NI heuristic is that it has the following 'recursive' property:

Property 3.1 The remaining part of the NI heuristic is equal to the NI heuristic for the rest of the items with the picker's current position as starting point.

To study the NI heuristic we will compare it with the Shorter Direction (SD) heuristic, which is described in Bartoldi and Platzman [1] as follows:

Step 1: Evaluate the length of the route that simply rotates clockwise, and the length of the route that simply rotates counter-clockwise.

Step 2: Choose the shorter of the two routes from step 1.

By applying the NI heuristic to retrieve a list of $n$ items, the picker will subsequently visit the positions $\omega_{i_{1}}, \omega_{i_{2}}, \ldots, \omega_{i_{n}}$. For convenience we denote

$$
x_{l}=\omega_{i_{l}}, l=1,2, \ldots, n ; x_{0}=\omega_{0} .
$$

We also introduce the following random variables: 
$T_{n}^{N I}$ - the travel time to retrieve $n$ items under the NI heuristic;

$T_{n}^{S D}$ - the travel time to retrieve $n$ items under the SD heuristic.

These random variables are of course functions of the elementary random event $\omega \epsilon$ $[0,1)^{n+1}$. Since the NI heuristic seems to be slightly more subtle than the SD heuristic, one may expect that it performs better with high probability. In fact, we will prove that the NI heuristic is never worse than the SD heuristic.

Lemma 3.2 For any $\omega \in[0,1)^{n+1}$ it holds that $T_{n}^{N I}(\omega) \leq T_{n}^{S D}(\omega)$.

Proof. We will present a proof by induction to $n$. It is clear that for any $\omega \in[0,1)^{2}$ we have $T_{1}^{N I}(\omega)=T_{1}^{S D}(\omega)=\rho\left(x_{0}, x_{1}\right)$. Now suppose that for some $n=1,2, \ldots$ we have $T_{n}^{N I}(\omega) \leq T_{n}^{S D}(\omega), \omega \in[0,1)^{n+1}$. Then we will prove that $T_{n+1}^{N I}(\omega) \leq T_{n+1}^{S D}(\omega)$, $\omega \in[0,1)^{n+2}$. The proof is illustrated in Fig. 2. First, recall that under the SD heuristic

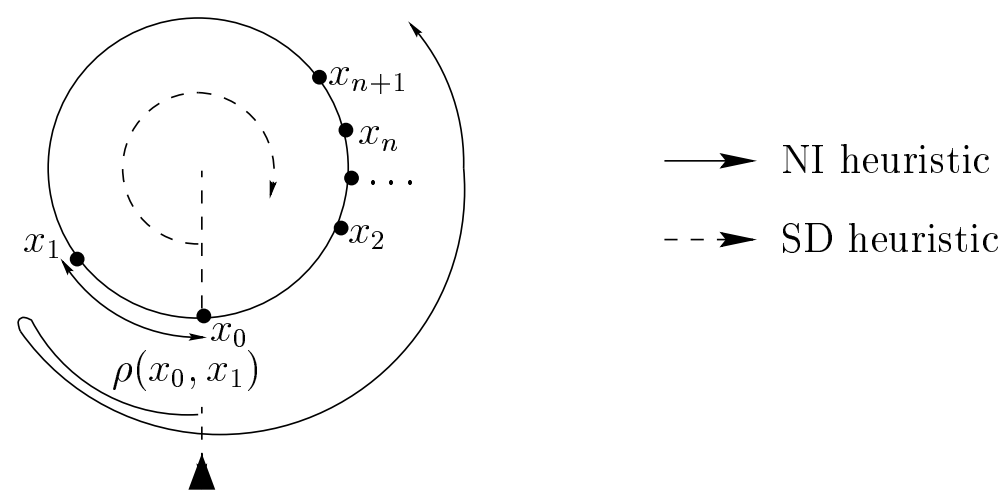

Figure 2: An illustration for the proof of Lemma 3.2.

the carousel always rotates in the same direction. There are only two possible routes of that kind, and their lengths differ only in the first segment. Therefore, choosing the shorter direction actually means choosing the shorter first interval. Hence, the algorithm for the SD heuristic can be formulated as follows:

Step 1: Rotate to the nearest item.

Step 2: Proceed further in the same direction.

It means that the NI and SD heuristic start with the same segment of length $\rho\left(x_{0}, x_{1}\right)$. After the first step the picker is at position $x_{1}$ and $n$ items remain to be picked. Thus, the current situation can be described by $\omega^{\prime} \in[0,1)^{n+1}$. The remaining travel time under the SD heuristic cannot be shorter than $T_{n}^{S D}\left(\omega^{\prime}\right)$, since by definition $T_{n}^{S D}\left(\omega^{\prime}\right)$ is the minimum travel time needed to pick $n$ items by proceeding in the same direction. Hence,

$$
\rho\left(x_{0}, x_{1}\right)+T_{n}^{S D}\left(\omega^{\prime}\right) \leq T_{n+1}^{S D}(\omega) .
$$


Further, due to property 3.1 we have

$$
T_{n+1}^{N I}(\omega)=\rho\left(x_{0}, x_{1}\right)+T_{n}^{N I}\left(\omega^{\prime}\right) .
$$

From (2), the induction assumption and (1) it follows that

$$
T_{n+1}^{N I}(\omega)=\rho\left(x_{0}, x_{1}\right)+T_{n}^{N I}\left(\omega^{\prime}\right) \leq \rho\left(x_{0}, x_{1}\right)+T_{n}^{S D}\left(\omega^{\prime}\right) \leq T_{n+1}^{S D}(\omega),
$$

which completes the proof.

In order to pick $n$ items under the NI heuristic, $n$ segments of the carousel should be covered. Their lengths are $\rho\left(x_{0}, x_{1}\right), \rho\left(x_{1}, x_{2}\right), \ldots, \rho\left(x_{n-1}, x_{n}\right)$. Note that they do not necessarily coincide with spacings between two adjacent items, since under the NI heuristic the carousel can rotate in different directions (see Fig. 2). Bartoldi and Platzman [1] showed that $T_{n}^{N I}$ is always less than 1 for all $n$. Now we will use Lemma 1 to prove the following stronger assertion.

Theorem 3.3 For any $\omega \in[0,1)^{n+1}$ and any $k=1,2, \ldots, n$, the total length of $k$ arbitrarily chosen segments that arise under the NI heuristic never exceeds $1-1 / 2^{k}$.

Proof. Consider the NI heuristics starting in an arbitrary point $x_{0} \in[0,1)$. Let $1 \leq l_{1}<l_{2}<\ldots<l_{k} \leq n$ be the indices of $k$ arbitrarily chosen segments in the order we cover them, and $\rho\left(x_{l_{1}-1}, x_{l_{1}}\right), \rho\left(x_{l_{2}-1}, x_{l_{2}}\right), \ldots, \rho\left(x_{l_{k}-1}, x_{l_{k}}\right)$ are their corresponding lengths.

We proceed with the NI heuristic until facing the first segment $l_{1}$. Now the picker is at point $x_{l_{1}-1}$, and there are still $n-l_{1}+1$ positions to be visited.

Consider the case that $\rho\left(x_{l_{1}-1}, x_{l_{1}}\right) \geq 1 / 2^{k}$. If we pick the remaining $n-l_{1}+1$ items under the SD heuristic starting at point $x_{l_{1}-1}$, then the travel time cannot exceed $1-1 / 2^{k}$. Then, from Property 3.1 and Lemma 3.2 it follows that the remaining travel time under the NI heuristic also does not exceed $1-1 / 2^{k}$. Recall that $l_{1}$ is the first one of the $k$ chosen segments faced under the NI heuristic. Hence, all $k$ segments under consideration are included in the remaining path. So, their total length cannot be greater than $1-1 / 2^{k}$.

Now, assume that $\rho\left(x_{l_{1}-1}, x_{l_{1}}\right)<1 / 2^{k}$. Then we proceed further until segment $l_{2}$ is faced. If $\rho\left(x_{l_{2}-1}, x_{l_{2}}\right) \geq 1 / 2^{k-1}$, then we can use similar arguments as above to conclude that the total length of the remaining $k-1$ of the $k$ chosen segments is not greater than $1-1 / 2^{k-1}$, and it immediately follows that the total length of $k$ chosen segments does not exceed

$$
\rho\left(x_{l_{1}-1}, x_{l_{1}}\right)+1-1 / 2^{k-1}<1 / 2^{k}+1-1 / 2^{k-1}=1-1 / 2^{k} .
$$

If $\rho\left(x_{l_{2}-1}, x_{l_{2}}\right)<1 / 2^{k-1}$, then we proceed with the NI heuristic and repeat the same arguments. Finally, two cases are possible:

1. There exists an $i=2,3, \ldots, k$ such that $\rho\left(x_{l_{j}-1}, x_{l_{j}}\right)<1 / 2^{k-j+1}, j=1,2, \ldots, i-1$, and $\rho\left(x_{l_{i}-1}, x_{l_{i}}\right) \geq 1 / 2^{k-i+1}$. In this case the remaining path under the NI heuristic 
is not longer than $1-1 / 2^{k-i+1}$, and therefore the total length of $k$ chosen segments does not exceed

$$
\begin{aligned}
\sum_{j=1}^{i-1} \rho\left(x_{l_{j}-1}, x_{l_{j}}\right)+1-\frac{1}{2^{k-i+1}} & <\frac{1}{2^{k}}+\frac{1}{2^{k-1}}+\ldots+\frac{1}{2^{k-i+2}}+1-\frac{1}{2^{k-i+1}} \\
& =1-\frac{1}{2^{k}} .
\end{aligned}
$$

2. For each $i=2,3, \ldots, k$ we have $\rho\left(x_{l_{i}-1}, x_{l_{i}}\right)<1 / 2^{k-i+1}$. Then the total length of the $k$ largest segments is less than

$$
\frac{1}{2^{k}}+\frac{1}{2^{k-1}}+\ldots+\frac{1}{2}=1-\frac{1}{2^{k}} .
$$

Thus, in both cases the assertion of the theorem holds.

Since the complete travel time is identical to the total length of the $n$ segments, an upper bound for the travel time under the NI heuristic immediately follows from Theorem 3.3.

Corollary 3.4 For each $\omega \in[0,1)^{n+1}$ the travel time under the NI heuristic satisfies

$$
T_{n}^{N I}(\omega) \leq 1-1 / 2^{n} .
$$

Let us give an example to show that Corollary 3.4 provides a tight upper bound.

Example 3.5 Let $n=5$, and let the starting position of the picker be $x_{0}=0$. The items to be picked are located at the positions $1 / 32,3 / 32,7 / 32,15 / 32$ and $31 / 32-\varepsilon$, where $\varepsilon$ is positive and arbitrarily small (see Fig. 3).

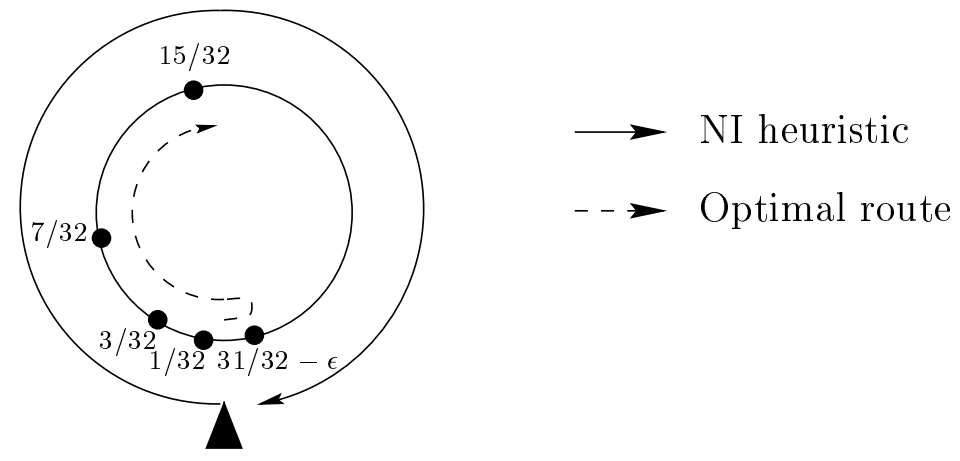

Figure 3: An example for which the travel time is arbitrarily close to the upper bound.

Then the travel distance under the NI heuristic is

$$
\frac{1}{32}+\frac{2}{32}+\frac{4}{32}+\frac{8}{32}+\left(\frac{16}{32}-\varepsilon\right)=\frac{31}{32}-\varepsilon=1-\frac{1}{2^{5}}-\varepsilon .
$$

The upper bound $1-1 / 2^{5}$ is tight, since $\varepsilon$ is arbitrarily small. A similar example can be easily constructed for any $n$. 
Remark 3.6 In Example 3.5 the travel time does not really achieve its upper bound. However, if the picker starts at point $x_{0}=0$ and needs to pick only one item at point $x_{1}=1 / 2$, or two items at points $x_{1}=1 / 4$ and $x_{2}=3 / 4$, then the travel time is equal to its upper bound (1/2 and 3/4 respectively). For $n>2$ the upper bound can also be achieved, if we assume that when the travel times to the nearest items clockwise and counter-clockwise are exactly the same, the picker always proceeds, say, clockwise. Now, if we put $\varepsilon=0$ in the example above, then the travel time will be exactly $1-1 / 2^{5}$.

Remark 3.7 Note that Example 3.5 is the only one we can construct to show that the upper bound is tight. Indeed, from the proof of Theorem 3.3 it follows that if the first segment is smaller or greater than $1 / 2^{n}$, then the travel time to pick $n$ items under the NI heuristic is less than $1-1 / 2^{n}$. The only case when the upper bound can be achieved is when $\rho\left(x_{0}, x_{1}\right)=1 / 2^{n}$. Then after the first step, the picker is at position $x_{1}$ and $n-1$ items remain to be picked. Due to Property 3.1 we can use similar arguments to show that the upper bound can only be achieved if $\rho\left(x_{1}, x_{2}\right)=1 / 2^{n-1}$. The same can be done for each of the $n$ steps under the NI heuristic. It implies that the upper bound can be achieved if and only if the $l$-th segment has length $1 / 2^{n-l+1}$ for all $l=1,2, \ldots, n$.

Fig. 3 also shows that the NI strategy is sometimes far from optimal. Indeed, in the case under consideration the optimal sequence is: $31 / 32-\varepsilon, 1 / 32,3 / 32,7 / 32,15 / 32$. The total length of this route is

$$
\left(\frac{1}{32}+\varepsilon\right)+\left(\frac{1}{32}+\varepsilon\right)+\frac{1}{32}+\frac{2}{32}+\frac{4}{32}+\frac{8}{32}=\frac{17}{32}+2 \varepsilon,
$$

which is much less than $31 / 32-\varepsilon$, when $\varepsilon$ is small.

\section{Mean travel time}

Let $U_{0: n+1}, U_{1: n+1}, \ldots, U_{n: n+1}$ denote the order statistics of the random variables $U_{0}, \ldots, U_{n}$ on $[0,1)$ (see Section 2). Then the random variables $D_{i}=U_{i: n+1}-U_{i-1: n+1}$ for $1 \leq i \leq n$ and $D_{n+1}=1-U_{n: n+1}+U_{0: n+1}$ are the spacings between two adjacent pick positions. To find the mean travel time under the NI heuristic we will use the following very useful property of these spacings. If $Y_{1}, \ldots, Y_{n+1}$ are independent exponentials with the same mean, then $\left(D_{1}, \ldots, D_{n+1}\right)$ is distributed as $\left(Y_{1} / \sum_{i=1}^{n+1} Y_{i}, \ldots, Y_{n+1} / \sum_{i=1}^{n+1} Y_{i}\right)$ (cf. Pyke [3, 4]). Hence the spacings are normalized exponentials.

Under the NI heuristic the picker does not have to know all spacings at once. He first considers the two spacings adjacent to his starting position and then moves to the nearest item. Next he also looks at the spacing adjacent to that item and moves again to the nearest item, and so on. Furthermore, note that we may act as if the picker faces nonnormalized exponential spacings, and afterwards divide the travel time by the sum of all spacings. Then it is clear that each new spacing faced by the picker is independent of the ones already observed. Now let $X_{i}$, where $i=1, \ldots, n+1$, denote the $i$-th non-normalized 


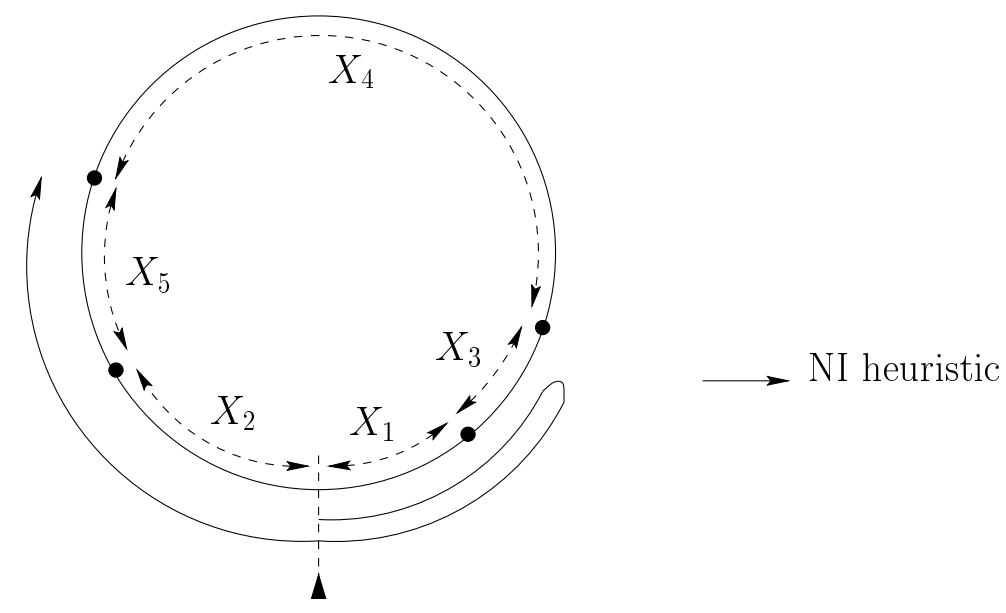

Figure 4: The NI route of the picker facing 5 exponential spacings.

exponential spacing faced by the picker. So the spacings are numbered as observed by the picker operating under the NI heuristic (see Fig. 4).

Then the travel time $T_{n}^{N I}$ can be expressed as

$$
T_{n}^{N I}=\sum_{i=1}^{n} \frac{\min \left(S_{i}, X_{i+1}\right)}{S_{n+1}},
$$

where $S_{i}=\sum_{j=1}^{i} X_{j}$. Hence by taking expectations we find

$$
E\left(T_{n}^{N I}\right)=\sum_{i=1}^{n} E\left(\frac{\min \left(S_{i}, X_{i+1}\right)}{S_{n+1}}\right) .
$$

The following lemma gives a simple formula for the expected travel time in the $i$-th step.

Lemma 4.1 Let $X_{1}, \ldots, X_{n+1}$ be $n+1$ independent exponentials with the same mean and let $S_{i}=\sum_{j=1}^{i} X_{j}, i=1, \ldots, n+1$. Then it holds that

$$
E\left(\frac{\min \left(S_{i}, X_{i+1}\right)}{S_{n+1}}\right)=\frac{1}{n+1}\left(1-\frac{1}{2^{i}}\right), \quad i=1, \ldots, n .
$$

Proof. Let $\mu$ denote the mean of each of the exponentials. Given the event

$$
E_{k}=\left[S_{k-1}<X_{i+1}<S_{k}\right],
$$

for some $k=1, \ldots, i$, the random variables $X_{1}, \ldots, X_{n+1}$ can be coupled as

$$
\begin{array}{ll}
X_{l}=\frac{1}{2} Y_{l}, \quad l=1, \ldots, k-1 ; & X_{k}=\frac{1}{2} Y_{k}+Y_{k+1} \\
X_{l}=Y_{l+1}, \quad l=k+1, \ldots, i ; & X_{i+1}=\sum_{l=1}^{k} \frac{1}{2} Y_{l} \\
X_{l}=Y_{l}, \quad l=i+2, \ldots, n+1, &
\end{array}
$$


where $Y_{1}, Y_{2}, \ldots$ are independent exponentials with mean $\mu$. This follows by observing that, given $E_{k}$, the random variable $X_{1}$ is the minimum of $X_{1}$ and $X_{i+1}$, and thus it is exponential with mean $\mu / 2$. Since the overshoot of $X_{i+1}$ is again exponential with mean $\mu$ we can repeat the argument for $X_{2}$ and so on. Eventually $X_{i+1}-S_{k-1}$ is less than $X_{k}$, so

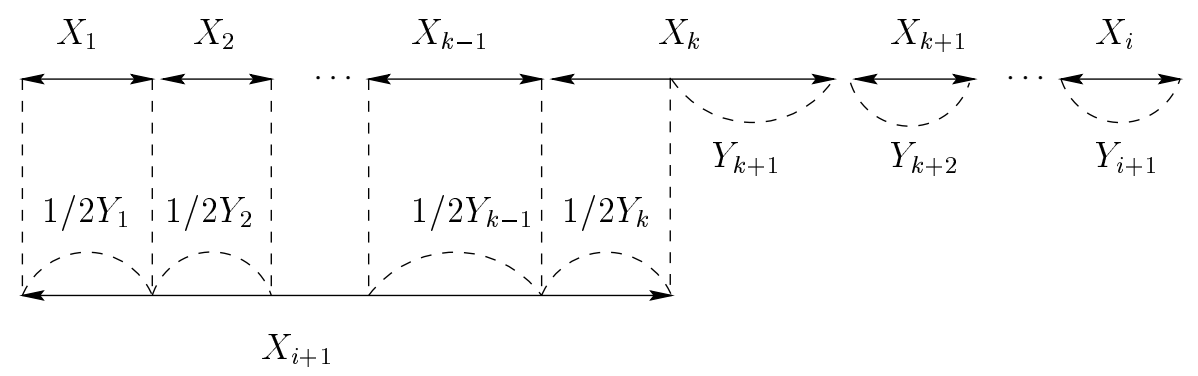

Figure 5: Coupling of the random variables $X_{1}, \ldots, X_{n+1}$ under event $E_{k}$.

it is exponential with mean $\mu / 2$. The random variable $X_{k}$ is the sum of two exponentials, one with mean $\mu / 2$ and the other part (i.e., the overshoot) with mean $\mu$ (see also Fig. 5). Since the event $E_{k}$ does not provide any information on the other random variables, they remain exponential with mean $\mu$.

Now, given the event $E_{k}$, it follows that

$$
\min \left(S_{i}, X_{i+1}\right)=X_{i+1}=\sum_{l=1}^{k} \frac{1}{2} Y_{l},
$$

and

$$
\begin{aligned}
S_{n+1} & =\frac{1}{2} Y_{1}+\cdots+\frac{1}{2} Y_{k}+Y_{k+1}+\cdots+Y_{i+1}+\sum_{l=1}^{k} \frac{1}{2} Y_{l}+Y_{i+2}+\cdots+Y_{n+1} \\
& =Y_{1}+\cdots+Y_{n+1} .
\end{aligned}
$$

So we obtain

$$
\begin{aligned}
E\left(\frac{\min \left(S_{i}, X_{i+1}\right)}{S_{n+1}} \mid E_{k}\right) & =E\left(\frac{\sum_{l=1}^{k} \frac{1}{2} Y_{l}}{Y_{1}+\cdots+Y_{n+1}}\right) \\
& =\frac{1}{2} \sum_{l=1}^{k} E\left(\frac{Y_{l}}{Y_{1}+\cdots+Y_{n+1}}\right)
\end{aligned}
$$

Since $Y_{1}, Y_{2}, \ldots$ are i.i.d., we have

$$
E\left(\frac{Y_{l}}{Y_{1}+\cdots+Y_{n+1}}\right)=\frac{1}{n+1}, \quad l=1, \ldots, n+1 .
$$

This immediately follows from the fact that these expectations are all the same and that they add up to 1 . Hence,

$$
E\left(\frac{\min \left(S_{i}, X_{i+1}\right)}{S_{n+1}} \mid E_{k}\right)=\frac{k}{2(n+1)} .
$$


Further, it is easily seen that

$$
E\left(\frac{\min \left(S_{i}, X_{i+1}\right)}{S_{n+1}} \mid X_{i+1}>S_{i}\right)=E\left(\frac{\min \left(S_{i}, X_{i+1}\right)}{S_{n+1}} \mid E_{i}\right) .
$$

Hence, since $\operatorname{Pr}\left[E_{k}\right]=1 / 2^{k}$, we find for the full expectation

$$
\begin{aligned}
E\left(\frac{\min \left(S_{i}, X_{i+1}\right)}{S_{n+1}}\right) & =\sum_{k=1}^{i} \frac{k}{2(n+1)} \cdot \frac{1}{2^{k}}+\frac{i}{2(n+1)} \cdot \frac{1}{2^{i}} \\
& =\frac{1}{n+1}\left(1-\frac{1}{2^{i}}\right)
\end{aligned}
$$

which completes the proof of the lemma.

From (3) and Lemma 4.1 we obtain after a simple calculation the following result.

Theorem 4.2 For all $n=1,2, \ldots$ we have:

$$
E\left(T_{n}^{N I}\right)=\frac{n}{n+1}-\left(1-\frac{1}{2^{n}}\right) \frac{1}{n+1} .
$$

Let us compare the mean performance of the NI and SD heuristics. One can verify (cf. Rouwenhorst et al. [5]) that

$$
P\left(T_{n}^{S D}<t\right)= \begin{cases}2 t^{n}, & 0 \leq t \leq 1 / 2 \\ 2 t^{n}-(2 t-1)^{n}, & 1 / 2<t \leq 1\end{cases}
$$

Hence, it is easy to compute that

$$
E\left(T_{n}^{S D}\right)=\frac{n}{n+1}-\frac{1}{2} \frac{1}{n+1} .
$$

If the carousel just rotates in the same arbitrarily chosen direction, then the mean travel time is clearly $n /(n+1)$, since there are $n$ segments to cover, and $1 /(n+1)$ is the average length of each segment. If the SD heuristic is applied, then the mean travel time will be reduced by $1 / 2$ of an average segment. By applying the NI heuristic, we can reduce the mean travel time by a fraction $1-1 / 2^{n}$ of an average segment. Obviously, when $n$ is large the difference between these different heuristics becomes negligible.

\section{Conditional mean travel time}

The probabilistic approach in the previous section may also be used for finding higher moments of the travel time under the NI heuristic, but here we will not elaborate this further. Instead, we expose in this section an alternative analytical approach to determine the mean travel time and in Section 6 we show that this approach is also suitable for finding higher moments. In fact, this approach yields more information than just the moments of 
the travel time, since it determines the moments of the remaining travel time after picking some items, i.e., the travel time conditioned on the size of the known empty space at one side of the picker's current position.

To derive a formula for the mean travel time under the NI heuristic we will develop a procedure exploiting property 3.1. According to this property the remaining part of the NI heuristic after the first step is equal to the NI heuristic for the other $n-1$ items with the picker's current position as starting point. The expected travel time of the first step can be found straightforwardly. However, the expectation of the remaining travel time is not just the mean travel time under the NI heuristic for $n-1$ items, because we also need to take into consideration the size of the empty space at one side of the picker's position. So, we can obtain a recursive equation for the mean travel time conditioned on the size of the empty space at one side of the picker's position. Denote by $E\left(T_{n}^{N I} \mid t\right)$ the mean travel time under the NI heuristic, given that at one side of the picker's starting point there is an empty space of size $t$. Then the mean travel time under the NI heuristic is just equal to $E\left(T_{n}^{N I} \mid 0\right)$ :

$$
E\left(T_{n}^{N I}\right)=E\left(T_{n}^{N I} \mid 0\right)
$$

Our object now is to derive a formula for $E\left(T_{n}^{N I} \mid t\right), 0 \leq t<1$.

The case $1 / 2 \leq t<1$ is trivial, since in this case the carousel will rotate in one direction only. It is easy to see that there are $n$ segments to cover, and the average length of each segment is $(1-t) /(n+1)$. Thus, we have:

$$
E\left(T_{n}^{N I} \mid t\right)=\frac{n}{n+1}(1-t), \quad 1 / 2 \leq t<1 .
$$

Let us now consider $0 \leq t<1 / 2$. We will derive a recursive equation for $E\left(T_{n}^{N I} \mid t\right)$ by conditioning on the location of the nearest item. Let $f_{n}(y \mid t)$ denote the density of the travel time to the nearest item given that there is an empty space of size $t$ near the starting point. There are two possible cases, which are shown in Fig. 6. For $y \leq t$ we have
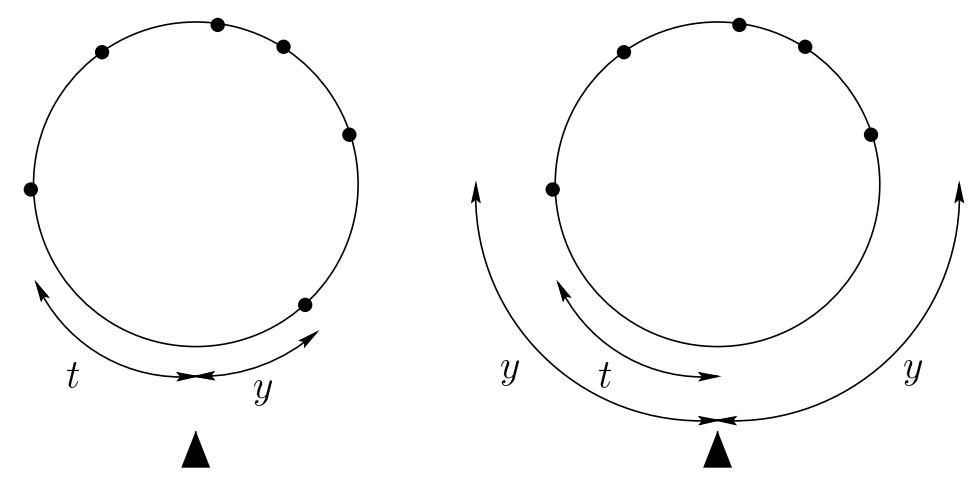

Figure 6: Two possible locations of the nearest item.

$$
f_{n}(y \mid t)=n(1-t-y)^{n-1} /(1-t)^{n}
$$


and after this step there will be an empty space of size $t+y$. For $t<y<1 / 2$ it holds that

$$
f_{n}(y \mid t)=2 n(1-2 y)^{n-1} /(1-t)^{n}
$$

and after such a step there will be an empty space of size $2 y$. Now we use the full expectation formula:

$$
\begin{aligned}
E\left(T_{n}^{N I} \mid t\right) & =\int_{0}^{t} \frac{n(1-t-y)^{n-1}}{(1-t)^{n}}\left[E\left(T_{n-1}^{N I} \mid t+y\right)+y\right] d y \\
& +\int_{t}^{1 / 2} \frac{2 n(1-2 y)^{n-1}}{(1-t)^{n}}\left[E\left(T_{n-1}^{N I} \mid 2 y\right)+y\right] d y, \quad 0 \leq t<1 / 2 .
\end{aligned}
$$

To find a solution for equation (6) we first introduce the functions

$$
D_{n}(t)=E\left(T_{n}^{N I} \mid t\right)(1-t)^{n}, \quad 0 \leq t<1
$$

Now we can rewrite equation (6) in the following form:

$$
\begin{aligned}
D_{n}(t) & =\int_{0}^{t} n D_{n-1}(t+y) d y+\int_{t}^{1 / 2} 2 n D_{n-1}(2 y) d y \\
& +\int_{0}^{t} n(1-t-y)^{n-1} y d y+\int_{t}^{1 / 2} 2 n(1-2 y)^{n-1} y d y, \quad 0 \leq t<1 / 2 .
\end{aligned}
$$

The last two integrals in (7) can be easily calculated, yielding

$$
\int_{0}^{t} n(1-t-y)^{n-1} y d y+\int_{t}^{1 / 2} 2 n(1-2 y)^{n-1} y d y={\frac{(1-t)^{n+1}}{n+1}}^{2(n+1)}-\frac{(1-2 t)}{n+1}^{n}
$$

Putting $\tau=y+t$ in the first integral and $\tau=2 y$ in the second one, we simplify equation $(7)$ to:

$$
D_{n}(t)=\int_{t}^{1} n D_{n-1}(\tau) d \tau+{\frac{(1-t)^{n+1}}{n+1}}^{n}-\frac{(1-2 t)}{2(n+1)}^{n+1} \quad, \quad 0 \leq t<1 / 2 .
$$

In this case the change of variables simplifies the recursion significantly. This simple trick will appear to be very helpful throughout the whole paper. However, as we will see in section 10, it does not always help that much. There we need to consider each of the intervals $0 \leq t<1 / 2^{n}, 1 / 2^{n} \leq t<1 / 2^{n-1}, \ldots, 1 / 4 \leq t<1 / 2$ separately, which makes the calculations much more complicated.

From (8) it is seen that one needs to know $D_{n-1}(t)$ at $1 / 2 \leq t<1$ to calculate $D_{n}(t)$ at $0 \leq t<1 / 2$. From (5) we have

$$
D_{n}(t)=\frac{n}{n+1}(1-t)^{n+1}, \quad 1 / 2 \leq t<1
$$

Since

$$
D_{0}(t)=E\left(T_{0}^{N I} \mid t\right)(1-t)^{0} \equiv 0
$$


the solution of (8) should be of the form

$$
D_{n}(t)=a_{n}(1-t)^{n+1}+b_{n}(1-2 t)^{n+1}, \quad 0 \leq t<1 / 2,
$$

where

$$
\begin{aligned}
& a_{n}=\frac{n}{n+1} a_{n-1}+\frac{1}{n+1} ; \quad a_{0}=0, \\
& b_{n}=\frac{n}{2(n+1)} b_{n-1}-\frac{1}{2(n+1)} ; \quad b_{0}=0 .
\end{aligned}
$$

Denoting $a_{n}^{\prime}=(n+1) a_{n}, b_{n}^{\prime}=(n+1) b_{n}$ we have:

$$
\begin{aligned}
& a_{n}^{\prime}=a_{n-1}^{\prime}+1=a_{0}^{\prime}+n=n \\
& b_{n}^{\prime}=\frac{1}{2} b_{n-1}^{\prime}-\frac{1}{2}=\frac{1}{2^{n}} b_{0}^{\prime}-\sum_{i=1}^{n} \frac{1}{2^{i}}=\frac{1}{2^{n}}-1
\end{aligned}
$$

which gives

$$
D_{n}(t)=\frac{n}{n+1}(1-t)^{n+1}-\frac{1}{n+1}\left(1-\frac{1}{2^{n}}\right)(1-2 t)^{n+1}, \quad 0 \leq t<1 / 2 .
$$

Function (11) satisfies both the recursion (8) and the initial condition $D_{0}(t)=0$.

Remark 5.1 We could immediately say that if $D_{n}(t)$ satisfies (10), then $a_{n}$ should necessarily be $n /(n+1)$. Otherwise, the function defined by (9) and (10) is not continuous at $t=1 / 2$.

Our results are summarized in the following theorem:

Theorem 5.2 For all $n=1,2, \ldots$ we have:

$$
E\left(T_{n}^{N I} \mid t\right)=\frac{n}{n+1}(1-t)-\left(1-\frac{1}{2^{n}}\right) \frac{(1-2 t)^{n+1}}{(n+1)(1-t)^{n}} \mathbf{1}_{[0 ; 1 / 2)}(t), \quad 0 \leq t<1 .
$$

Of course, when we set $t=0$ in (12) we retrieve formula (4) for the (unconditional) mean travel time. Below in figure 7 we show the conditional expectation of the travel time as a function of the empty space $t$ for $n$ equal to 2, 5 and 10. Surprisingly, we see that the graphs slightly increase for small $t$. This is very well seen for $n=2$. It means that information about the empty space can be 'negative'. This may be explained by the fact that this information reduces the probability that items must be retrieved nearby the picker's position. Another observation is that the conditional expectation tends very fast to a linear function, which is of course also apparent from formula (12). 


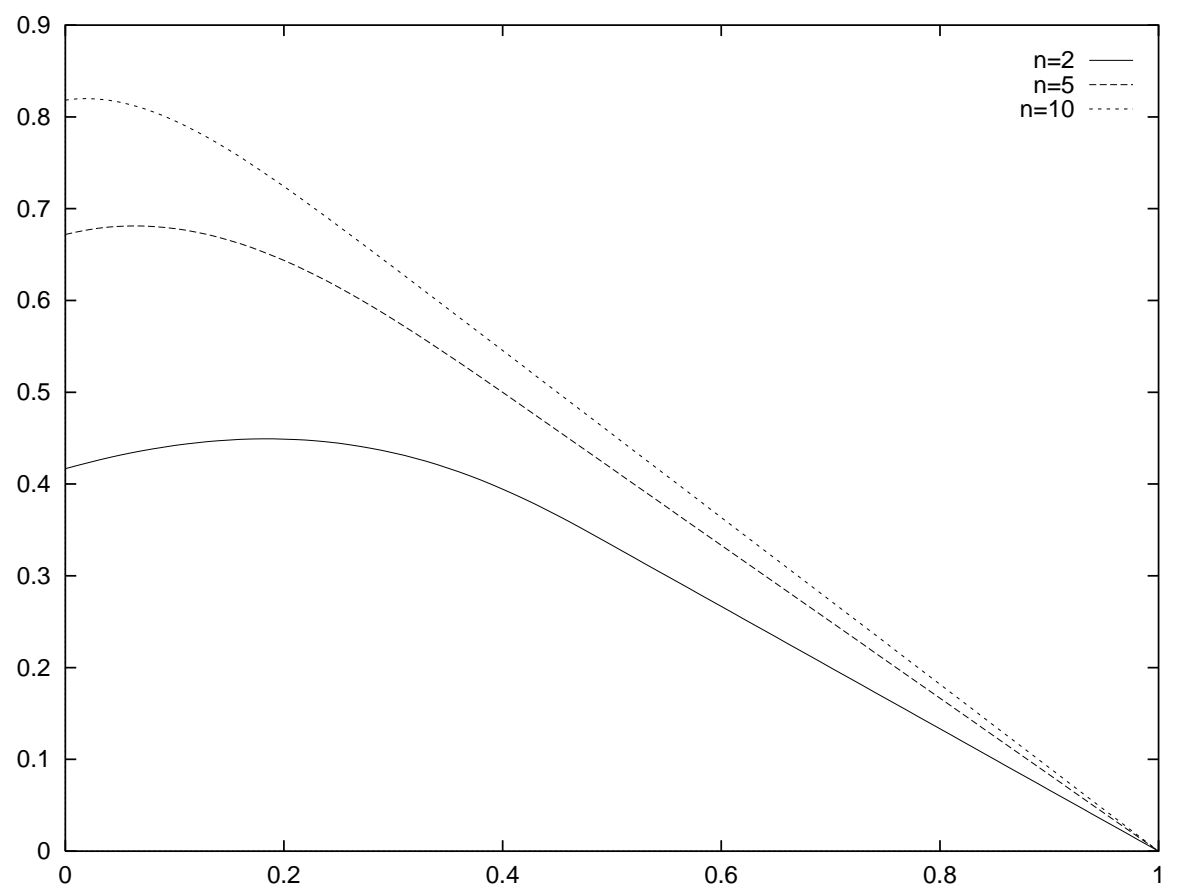

Figure 7: The conditional mean travel time as a function of the empty space $t$.

\section{Variance of the travel time}

The power of the analytical approach in the previous section is that it can also be used to obtain higher moments of $T_{n}^{N I}$. For example, for the second moment we need to consider the conditional expectation $E\left(\left[T_{n}^{N I}\right]^{2} \mid t\right)$. One can easily see that

$$
E\left(\left[T_{n}^{N I}\right]^{2} \mid t\right)=\frac{n}{n+2}(1-t)^{2}, \quad 1 / 2 \leq t<1,
$$

and that a recursive equation similar to (6) holds for $0 \leq t<1 / 2$ :

$$
\begin{aligned}
E\left(\left[T_{n}^{N I}\right]^{2} \mid t\right) & =\int_{0}^{t} \frac{n(1-t-y)^{n-1}}{(1-t)^{n}} E\left(\left[T_{n-1}^{N I}+y\right]^{2} \mid t+y\right) d y \\
& +\int_{t}^{1 / 2} \frac{2 n(1-2 y)^{n-1}}{(1-t)^{n}} E\left(\left[T_{n-1}^{N I}+y\right]^{2} \mid 2 y\right) d y, \quad 0 \leq t<1 / 2 .
\end{aligned}
$$

By introducing the functions

$$
D_{n}^{(2)}(t)=E\left(\left[T_{n}^{N I}\right]^{2} \mid t\right)(1-t)^{n}
$$

and changing variables, we can rewrite (14) in the following form:

$$
D_{n}^{(2)}(t)=\int_{t}^{1} n D_{n-1}^{(2)}(\tau) d \tau
$$




$$
\begin{aligned}
& +\int_{0}^{t} 2 n y D_{n-1}(t+y) d y+\int_{t}^{1 / 2} 4 n y D_{n-1}(2 y) d y \\
& +\int_{0}^{t} n y^{2}(1-t-y)^{n-1} d y+\int_{t}^{1 / 2} 2 n y^{2}(1-2 y)^{n-1} d y, \quad 0 \leq t<1 / 2 .
\end{aligned}
$$

Substituting (9) and (11) into (15) for $1 / 4 \leq t<1 / 2$ we obtain

$$
\begin{aligned}
D_{n}^{(2)}(t) & =\int_{t}^{1} n D_{n-1}^{(2)}(\tau) d \tau+\frac{2 n(1-t)^{n+2}}{(n+1)(n+2)} \\
& -\frac{t(1-2 t)^{n+1}}{n+1}-\left(n+\left(1-\frac{1}{2^{n}}\right)\right) \frac{(1-2 t)^{n+2}}{(n+1)(n+2)},
\end{aligned}
$$

and for $0 \leq t<1 / 4$ we get

$$
\begin{aligned}
D_{n}^{(2)}(t) & =\int_{t}^{1} n D_{n-1}^{(2)}(\tau) d \tau+\frac{2 n(1-t)^{n+2}}{(n+1)(n+2)}-\frac{t(1-2 t)^{n+1}}{n+1} \\
& -\left(n+\left(1-\frac{1}{2^{n}}\right)\right) \frac{(1-2 t)^{n+2}}{(n+1)(n+2)}+\frac{(1-4 t)^{n+2}}{4(n+1)(n+2)}\left(1-\frac{1}{2^{n-1}}\right) .
\end{aligned}
$$

From (13) we know that

$$
D_{n}^{(2)}(t)=\frac{n}{n+2}(1-t)^{n+2}, \quad 1 / 2 \leq t<1 .
$$

Thus, we can first solve the recursion (16) and then (17) exactly as it was done before. However, the calculations become much more complicated. Note that our method performs the calculations 'backwards' (see also Remark 6.3). This finally leads to the following result.

Theorem 6.1 For all $n=1,2, \ldots$ and $0 \leq t<1$ we have:

$$
\begin{aligned}
E\left(\left[T_{n}^{N I}\right]^{2} \mid t\right) & =\frac{n}{n+2}(1-t)^{2}-\frac{2}{n+1}\left(1-\frac{1}{2^{n}}\right) \frac{t(1-2 t)^{n+1}}{(1-t)^{n}} \mathbf{1}_{[0 ; 1 / 2)}(t) \\
& -\frac{2 n+1}{(n+1)(n+2)}\left(1-\frac{1}{2^{n}}\right) \frac{(1-2 t)^{n+2}}{(1-t)^{n}} \mathbf{1}_{[0 ; 1 / 2)}(t) \\
& +\frac{1}{(n+1)(n+2)}\left(\frac{1}{3}-\frac{1}{2^{n}}+\frac{2}{3 \cdot 4^{n}}\right) \frac{(1-4 t)^{n+2}}{(1-t)^{n}} \mathbf{1}_{[0 ; 1 / 4)}(t),
\end{aligned}
$$

Corollary 6.2 For all $n=1,2, \ldots$ it holds that:

$$
\begin{aligned}
& E\left(\left[T_{n}^{N I}\right]^{2}\right)=\frac{1}{(n+1)(n+2)}\left(n^{2}-n-\frac{2}{3}+\frac{n}{2^{n-1}}+\frac{2}{3 \cdot 4^{n}}\right) \\
& \operatorname{Var}\left(T_{n}^{N I}\right)=\frac{1}{(n+1)^{2}(n+2)}\left(\frac{4 n}{3}-\frac{n}{3 \cdot 4^{n}}-\frac{8}{3}+\frac{1}{2^{n-2}}-\frac{1}{3 \cdot 4^{n-1}}\right) .
\end{aligned}
$$

Remark 6.3 Our method determines the second moment $D_{n}^{(2)}(t)$ according to a backward recursion: it subsequently solves $D_{n}^{(2)}(t)$ on the intervals $[1 / 2,1),[1 / 4,1 / 2)$ and $[0,1 / 4)$. To determine the $k$-th moment we will have to consider the sequence of the intervals $[1 / 2,1),[1 / 4,1 / 2), \ldots,\left[0,1 / 2^{k}\right)$. 


\section{Approximation for the distribution function}

In this section we introduce a simple two-moment approximation for the distribution function of the travel time under the NI heuristic. Namely, we will compare the distribution of $T_{n}^{N I}$ with the distribution of the random value $X_{n}$, given by $X_{n}=u_{n} Y_{n}$, where the scaling factor $u_{n}$ is given by

$$
u_{n}=1-\frac{1}{2^{n}}
$$

and $Y_{n}$ has a beta-density, i.e.,

$$
f_{Y_{n}}(x)=\frac{\Gamma\left(\mu_{n}+\nu_{n}\right)}{\Gamma\left(\mu_{n}\right) \Gamma\left(\nu_{n}\right)}(1-x)^{\mu_{n}-1} x^{\nu_{n}-1}, \quad 0<x<1 .
$$

Clearly, the random variables $X_{n}$ and $T_{n}^{N I}$ have the same support (see Corollary 3.4) and their first two moments match if we set

$$
\begin{aligned}
\mu_{n} & =\frac{\left(u_{n}-E\left(T_{n}^{N I}\right)\right)\left(E\left(T_{n}^{N I}\right)-E\left(\left[T_{n}^{N I}\right]^{2}\right) / u_{n}\right)}{\operatorname{Var}\left(T_{n}^{N I}\right)}, \\
\nu_{n} & =\frac{E\left(T_{n}^{N I}\right)\left(E\left(T_{n}^{N I}\right)-E\left(\left[T_{n}^{N I}\right]^{2}\right) / u_{n}\right)}{\operatorname{Var}\left(T_{n}^{N I}\right)}
\end{aligned}
$$

Numerical results suggest that the approximation is quite accurate. Fig. 8 shows the

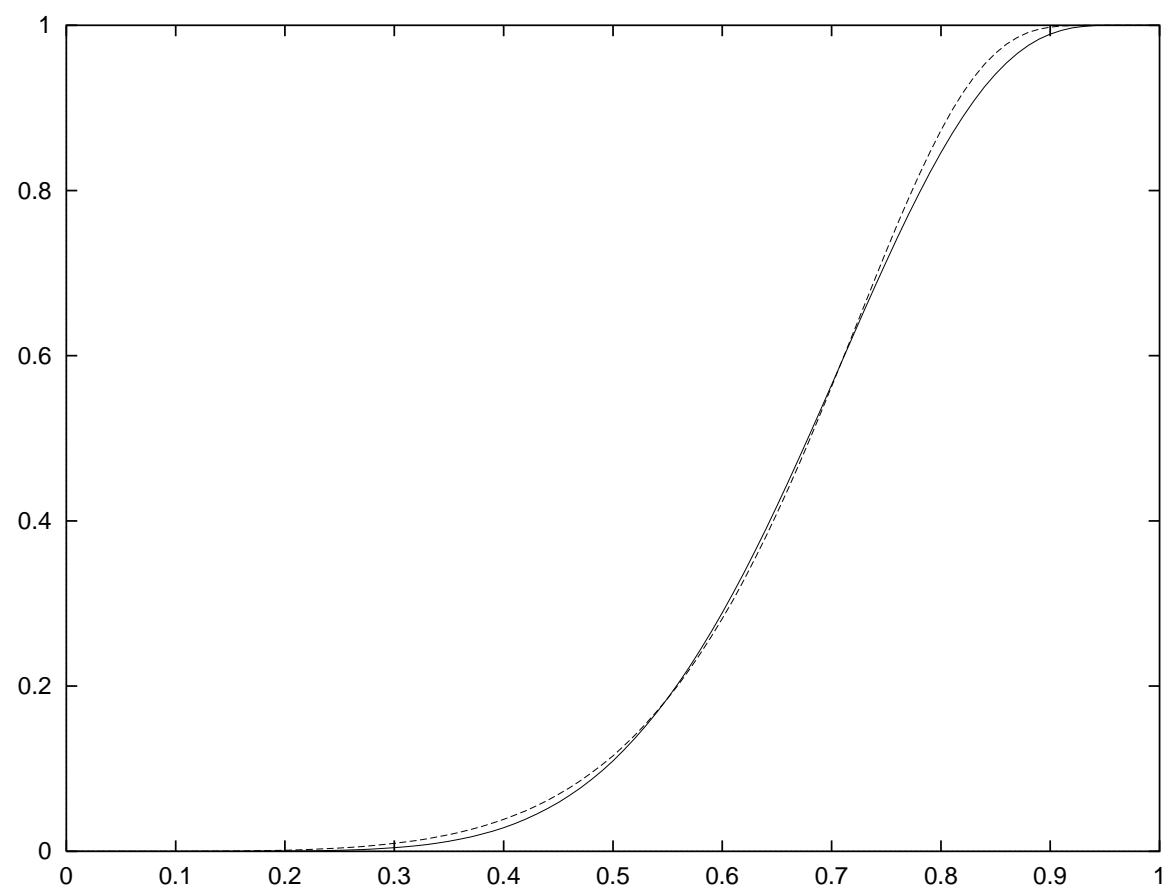

Figure 8: The distribution functions of $X_{5}$ (solid) and $T_{5}^{N I}$ (dashed).

distribution function of $X_{5}$ and the empirical distribution function of $T_{5}^{N I}$ obtained by a simulation of $10^{6}$ trials. Here the maximal absolute difference between the two distribution functions is about 0.03. An error of the same order occurs for all $n \geq 2$. 


\section{Distribution of the number of turns}

In this section we will determine the distribution of the number of turns under the NI heuristic. Let the random variable $K_{n}$ denote the number of turns to pick $n$ items under the NI heuristic, and define

$$
T_{i}=\mathbf{1}_{\left[S_{i}<X_{i+1}\right]}, \quad i=1,2, \ldots,
$$

where $X_{1}, X_{2}, \ldots$ are independent exponentials with the same mean, $\mu$ say, and $S_{i}=$ $\sum_{j=1}^{i} X_{j}, i \geq 1$. Since $X_{i}$ can be interpreted as the $i$-th non-normalized exponential spacing faced by the picker (see Section 4 ), it is clear that the random variable $T_{i}$ indicates whether or not the picker turns after picking the $(i-1)$-th item. So we may write

$$
K_{n}=\sum_{i=2}^{n} T_{i}
$$

Since $E\left(T_{i}\right)=\operatorname{Pr}\left[S_{i}<X_{i+1}\right]=1 / 2^{i}$ we immediately obtain the following result.

Theorem 8.1 For all $n=1,2, \ldots$ we have:

$$
E\left(K_{n}\right)=\frac{1}{2}-\frac{1}{2^{n}}
$$

To find the variance, and in fact, the complete distribution of the number of turns, we need the following remarkable result.

Lemma 8.2 The random variables $T_{1}, T_{2}, \ldots$ are independent.

Proof. We will prove that each pair is independent. The independence of any finite sequence of these random variables can be proved along the same lines. Let $1 \leq i<j$. To prove that $T_{i}$ and $T_{j}$ are independent, it suffices to show that

$$
\operatorname{Pr}\left[S_{i}<X_{i+1}, S_{j}<X_{j+1}\right]=\operatorname{Pr}\left[S_{i}<X_{i+1}\right] \cdot \operatorname{Pr}\left[S_{j}<X_{j+1}\right]
$$

Clearly,

$$
\operatorname{Pr}\left[S_{i}<X_{i+1}, S_{j}<X_{j+1}\right]=\operatorname{Pr}\left[S_{i}<X_{i+1}\right] \cdot \operatorname{Pr}\left[S_{j}<X_{j+1} \mid S_{i}<X_{i+1}\right] .
$$

Now, given the event $E_{i}=\left[S_{i}<X_{i+1}\right]$, we can couple $X_{1}, \ldots, X_{j+1}$ in the same way as done in the proof of Lemma 4.1, i.e.,

$$
\begin{aligned}
& X_{l}=\frac{1}{2} Y_{l}, \quad l=1, \ldots, i ; \quad X_{i+1}=\sum_{l=1}^{i} \frac{1}{2} Y_{l}+Y_{i+1} ; \\
& X_{l}=Y_{l}, \quad l=i+2, \ldots, j+1,
\end{aligned}
$$


where $Y_{1}, Y_{2}, \ldots$ are independent exponentials with mean $\mu$. Hence, given event $E_{i}$, we have $X_{j+1}=Y_{j+1}$ and

$$
\begin{aligned}
S_{j} & =\frac{1}{2} Y_{1}+\cdots+\frac{1}{2} Y_{i}+\sum_{l=1}^{i} \frac{1}{2} Y_{l}+Y_{i+1}+Y_{i+2}+\cdots+Y_{j} \\
& =Y_{1}+\cdots+Y_{j} .
\end{aligned}
$$

So

$$
\operatorname{Pr}\left[S_{j}<X_{j+1} \mid S_{i}<X_{i+1}\right]=\operatorname{Pr}\left[Y_{1}+\cdots+Y_{j}<Y_{j+1}\right]
$$

which, together with (20), proves equality (19).

Remark 8.3 The above lemma is not valid for, e.g., uniform random variables $X_{1}, X_{2}, \ldots$ on the interval $(0,1)$. It is easily verified that in this case we have

$$
\operatorname{Pr}\left[S_{2}<X_{3}, S_{3}<X_{4}\right]=\frac{1}{96}
$$

and

$$
\operatorname{Pr}\left[S_{2}<X_{3}\right]=\frac{1}{6}, \quad \operatorname{Pr}\left[S_{3}<X_{4}\right]=\frac{1}{24},
$$

so equality (19) does not hold.

From (18) and Lemma 8.2 we obtain for the second moment that

$$
\begin{aligned}
E\left(T_{n}^{2}\right) & =\sum_{i=2}^{n} E\left(T_{i}^{2}\right)+\sum_{2 \leq i<j \leq n} 2 E\left(T_{i} T_{j}\right) \\
& =\sum_{i=2}^{n} E\left(T_{i}\right)+\sum_{2 \leq i<j \leq n} 2 E\left(T_{i}\right) E\left(T_{j}\right) \\
& =\frac{1}{2}-\frac{1}{2^{n}}+\sum_{2 \leq i<j \leq n} \frac{1}{2^{i+j-1}} \\
& =\frac{2}{3}-\frac{1}{2^{n-1}}+\frac{1}{3 \cdot 4^{n-1}} .
\end{aligned}
$$

This yields the following formula for the variance of the number of turns.

Theorem 8.4 For all $n=1,2, \ldots$ we have:

$$
\operatorname{Var}\left(K_{n}\right)=\frac{5}{12}-\frac{1}{2^{n}}+\frac{1}{3 \cdot 4^{n}}
$$

Of course, from Lemma 8.2 we can also obtain the distribution of the number of turns: 
Theorem 8.5 For all $0 \leq k<n$ we have:

$$
\operatorname{Pr}\left[K_{n}=k\right]=\sum_{\substack{0 \leq k_{2}, \ldots, k_{n} \leq 1 \\ k_{2}+\cdots+k_{n}=k}} \operatorname{Pr}\left[T_{2}=k_{2}\right] \cdots \operatorname{Pr}\left[T_{n}=k_{n}\right],
$$

where

$$
\operatorname{Pr}\left[T_{i}=1\right]=1-\operatorname{Pr}\left[T_{i}=0\right]=\frac{1}{2^{i}}, \quad i=2, \ldots, n
$$

For computational purposes we mention that the probability distribution for $K_{n}$ can be determined recursively. Let $p_{n, k}$ denote $\operatorname{Pr}\left[K_{n}=k\right]$. From Theorem 8.5 we then obtain, by conditioning on $T_{n}$, the following recursion:

$$
p_{n, k}=\frac{1}{2^{n}} p_{n-1, k-1}+\left(1-\frac{1}{2^{n}}\right) p_{n-1, k}, \quad 0 \leq k<n,
$$

with initial condition $p_{1,0}=1$ and boundary conditions $p_{n,-1}=p_{n, n}=0$ for $n>1$. In Fig. 9 we show the distribution for $n=4,5,7$ and 10 . We see that it rapidly converges to

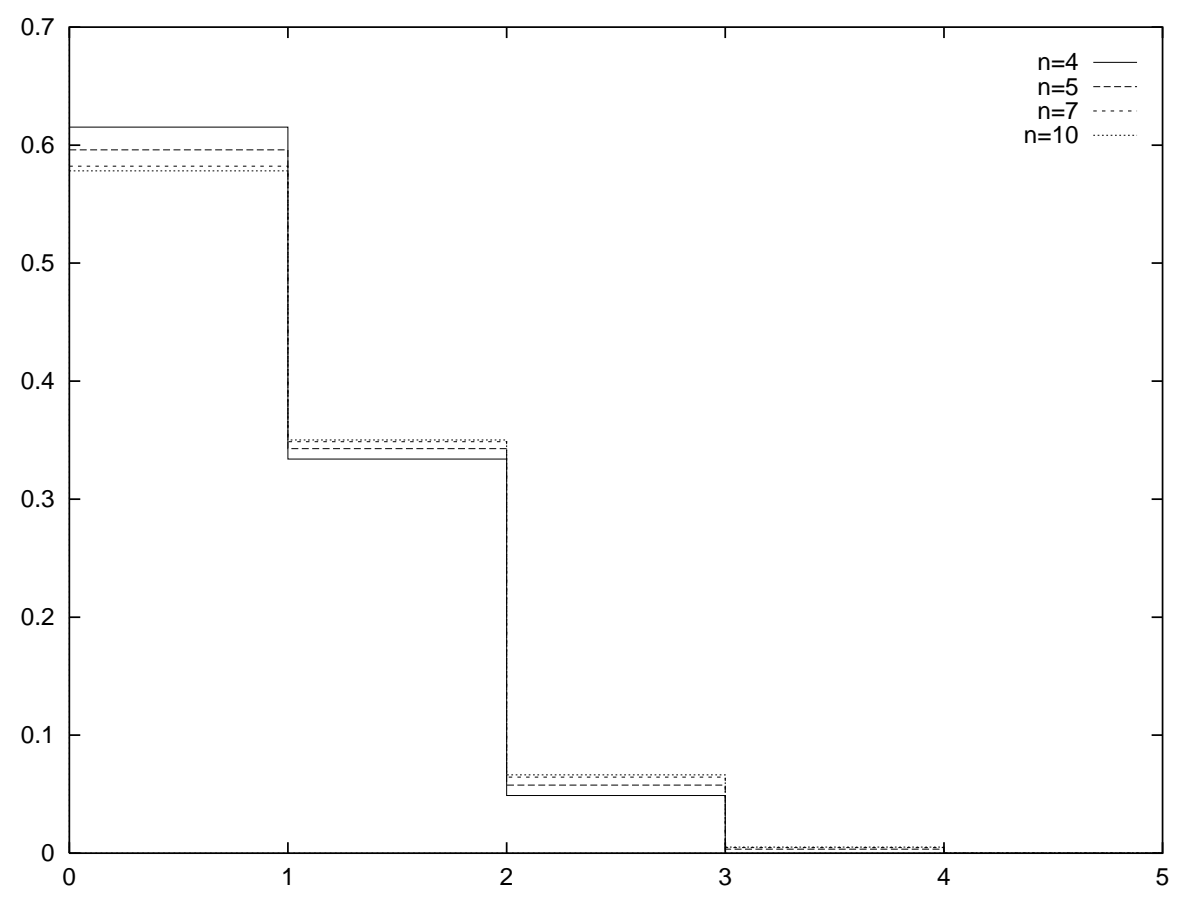

Figure 9: Distribution for the number of turns.

the limiting distribution for $K_{n}$ as $n \rightarrow \infty$. Let the random variable $K_{\infty}$ have this limiting distribution. From Theorem 8.5 we directly obtain

$$
\operatorname{Pr}\left[K_{\infty}=0\right]=\prod_{i=2}^{\infty}\left(1-\frac{1}{2^{i}}\right) \approx 0.5776,
$$




$$
\begin{aligned}
& \operatorname{Pr}\left[K_{\infty}=1\right]=\prod_{i=2}^{\infty}\left(1-\frac{1}{2^{i}}\right) \sum_{k=2}^{\infty} \frac{1}{2^{k}-1} \approx 0.3504 \\
& \operatorname{Pr}\left[K_{\infty}=2\right]=\prod_{i=2}^{\infty}\left(1-\frac{1}{2^{i}}\right) \sum_{2 \leq k<l}^{\infty} \frac{1}{2^{k}-1} \cdot \frac{1}{2^{l}-1} \approx 0.0666
\end{aligned}
$$

and so on. An alternative and elegant expression for the distribution of $K_{\infty}$ will be derived in Section 10. Note that the NI heuristic with no turns is actually the SD heuristic. One might intuitively expect that for large $n$ it is very unlikely that the carousel will change direction. However, we see that the probability that the NI and SD heuristics coincide does not tend to 1 as $n \rightarrow \infty$, but it decreases to approximately 0,5776 . So, in the limit the NI heuristic oscillates with quite high probability. However, the oscillation is very modest, since the limiting probability of 4 turns is about 0.0002 , and the probability of more than 4 turns is negligible.

\section{Conditional mean and variance for the number of turns}

In this section we first derive an upper bound for the number of remaining turns after picking the $i$-th item. Next we will obtain the mean and variance of the number of remaining turns conditioned on an empty space of size $t$ at one side of the picker's position.

Bartoldi and Platzman [1] mention that a route to pick $n$ items under the NI heuristic actually consists of a number of segments of uninterrupted clockwise and counterclockwise movements. Denote the number of segments by $N$ (so the number of turns is $N-1$ ) and let $I_{j}$ denote the length along the $j$-th segment to the first item retrieved on that segment (see Fig 10). Then they notice that

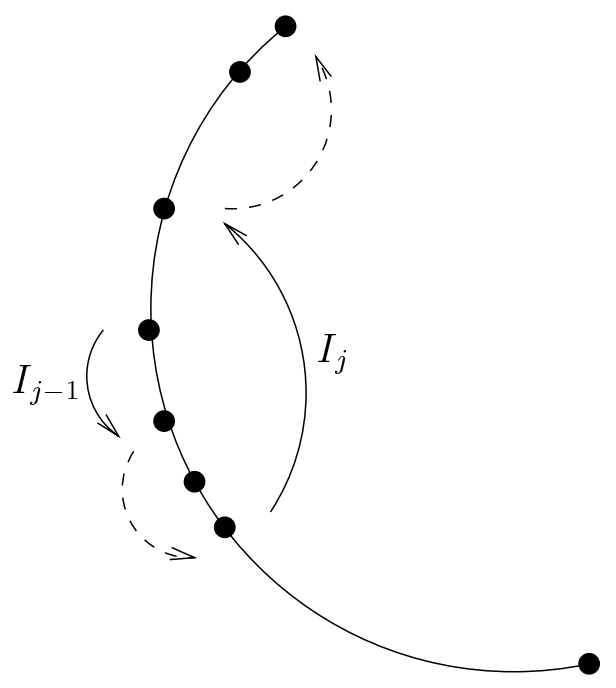

Figure 10: Segments of uninterrupted clockwise and counterclockwise movements. 


$$
I_{j} \geq 2 I_{j-1}, \quad j=2, \ldots, N \text {. }
$$

From this observation it immediately follows that

$$
I_{1} \leq 1 / 2 I_{2} \leq \ldots \leq 1 / 2^{N-1} I_{N} \leq 1 / 2^{N}
$$

Here the last inequality follows from the simple fact that any step under the NI heuristic is never greater than $1 / 2$. Thus we have proved the following lemma, which allows us to estimate the number of remaining turns after picking the $i$-th item:

Lemma 9.1 The number of remaining turns under the NI heuristic after picking the $i$-th item in the $j$-th segment, $1 \leq j \leq i \leq n$, is never greater than ${ }^{1 / 2} \log I_{j}-1$.

Now we will use the procedure from the Sections 5 and 6 to obtain the mean and variance of the number of turns conditioned on an empty space of size $t$ at one side of the picker's position. Let $E\left(K_{n} \mid t\right)$ be the expected number of turns conditioned on the empty space $t$. If the size of the empty space is greater than $1 / 2$, then no turns are possible:

$$
E\left(K_{n} \mid t\right)=0, \quad 1 / 2 \leq t<1 .
$$

For $t<1 / 2$ note that changing direction actually implies crossing the known empty space. The probability of this event is

$$
\operatorname{Pr}[\operatorname{crossing} \text { an empty space of size } t]=\int_{t}^{1 / 2} \frac{n(1-2 y)^{n-1}}{(1-t)^{n}} d y=\frac{(1-2 t)^{n}}{2(1-t)^{n}} \text {. }
$$

However, if $t=0$, then this probability becomes $1 / 2$. This is in contradiction with the natural assumption that the first step is never a turn. Hence, the case $t=0$ becomes exceptional. To avoid that we introduce an artificial random variable

$$
K_{n}^{\prime}=K_{n}+Z
$$

where $Z$ is a random variable independent of $K_{n}$, and

$$
\begin{aligned}
\operatorname{Pr}[Z=0 \mid t] & =0, \quad 0<t<1, \\
\operatorname{Pr}[Z=0 \mid 0] & =\operatorname{Pr}[Z=1 \mid 0]=1 / 2 .
\end{aligned}
$$

The conditional characteristics of $K_{n}^{\prime}$ are continuous at $t=0$, and for $0<t<1$ they coincide with conditional characteristics of $K_{n}$. Thus, we are first going to find the conditional mean, variance and (in Section 10) the conditional distribution of $K_{n}^{\prime}$. Then we can retrieve formulas for the mean, variance and distribution of $K_{n}$, by putting $t=0$ and applying (25).

We use $K_{n}^{\prime}$ mostly as an auxiliary random variable. However, it has a reasonable interpretation itself. Indeed, the picker's starting point is often just the last point of the previous order, and the picker reaches this point following a certain direction. To pick the first item of the next order the picker changes the direction with probability $1 / 2$. If this 
event is also considered as a turn, then the total number of turns is actually distributed as $K_{n}^{\prime}\left(\right.$ instead of $\left.K_{n}\right)$.

For the conditional expectation $E\left(K_{n}^{\prime} \mid t\right)$, where $0 \leq t<1 / 2$, we have the recursion

$$
\begin{aligned}
E\left(K_{n}^{\prime} \mid t\right) & =\int_{0}^{t} \frac{n(1-t-y)^{n-1}}{(1-t)^{n}} E\left(K_{n-1}^{\prime} \mid t+y\right) d y \\
& +\int_{t}^{1 / 2} \frac{n(1-2 y)^{n-1}}{(1-t)^{n}} E\left(K_{n-1}^{\prime} \mid 2 y\right) d y \\
& +\int_{t}^{1 / 2} \frac{n(1-2 y)^{n-1}}{(1-t)^{n}}\left[E\left(K_{n-1}^{\prime} \mid 2 y\right)+1\right] d y, \quad 0 \leq t<1 / 2 .
\end{aligned}
$$

Denoting

$$
C_{n}(t)=E\left(K_{n}^{\prime} \mid t\right)(1-t)^{n},
$$

and taking into consideration (24) we can rewrite (26) as

$$
C_{n}(t)=\int_{t}^{1 / 2} n C_{n-1}(\tau) d \tau+\frac{(1-2 t)^{n}}{2} .
$$

This recursions can be solved in the same way as done in the Sections 5 and 6 . The outcome is presented in the following theorem.

Theorem 9.2 For all $n=1,2, \ldots$ we have:

$$
E\left(K_{n}^{\prime} \mid t\right)=\left(1-\frac{1}{2^{n}}\right) \frac{(1-2 t)^{n}}{(1-t)^{n}} \mathbf{1}_{[0 ; 1 / 2)}(t), \quad 0 \leq t<1 .
$$

Formula (18) from Theorem 8.1 can now be obtained as follows:

$$
E\left(K_{n}\right)=E\left(K_{n}^{\prime} \mid 0\right)-E(Z \mid 0)=\frac{1}{2}-\frac{1}{2^{n}} .
$$

For the conditional second moment $E\left(\left[K_{n}^{\prime}\right]^{2} \mid t\right)$ we again apply the same procedure as for $E\left(\left[T_{n}^{N I}\right]^{2} \mid t\right)$ in Section 6. This yields

Theorem 9.3 For all $n=1,2, \ldots$ we have:

$$
\begin{aligned}
E\left(\left[K_{n}^{\prime}\right]^{2} \mid t\right) & =\left(1-\frac{1}{2^{n}}\right) \frac{(1-2 t)^{n}}{(1-t)^{n}} \mathbf{1}_{[0 ; 1 / 2)}(t) \\
& +2\left(\frac{1}{3}-\frac{1}{2^{n}}+\frac{2}{3 \cdot 4^{n}}\right) \frac{(1-4 t)^{n}}{(1-t)^{n}} \mathbf{1}_{[0 ; 1 / 4)}(t), \quad 0 \leq t<1 .
\end{aligned}
$$

From Theorem 9.3 it follows that

$$
\operatorname{Var}\left(K_{n}\right)=\operatorname{Var}\left(K_{n}^{\prime} \mid 0\right)-\operatorname{Var}(Z \mid 0)=\frac{5}{12}-\frac{1}{2^{n}}+\frac{1}{3 \cdot 4^{n}},
$$

which coincides with (21) from Theorem 8.4. 


\section{Conditional distribution for the number of turns}

Using the recursive procedure, we can also derive the conditional distribution for the random variable $K_{n}^{\prime}$. Let $\operatorname{Pr}\left[K_{n}^{\prime}=k \mid t\right]$ be the probability that $K_{n}^{\prime}$ equals $k$, if there is an empty space of size $t$ at one side of the picker's position. We will first determine $\operatorname{Pr}\left[K_{n}^{\prime}=0 \mid t\right]$. Clearly,

$$
\operatorname{Pr}\left[K_{n}^{\prime}=0 \mid t\right]=1, \quad 1 / 2 \leq t<1
$$

Further, we obtain

$$
\begin{aligned}
\operatorname{Pr}\left[K_{n}^{\prime}=0 \mid t\right] & =\int_{0}^{t} \mathrm{P}\left(K_{n-1}^{\prime}=0 \mid t+y\right) \frac{n(1-t-y)^{n-1}}{(1-t)^{n}} d y \\
& +\int_{t}^{1 / 2} \mathrm{P}\left(K_{n-1}^{\prime}=0 \mid 2 y\right) \frac{n(1-2 y)^{n-1}}{(1-t)^{n}} d y, \quad 0 \leq t<1 / 2 .
\end{aligned}
$$

By introducing

$$
L_{n}^{(0)}(t)=\mathrm{P}\left(K_{n}^{\prime}=0 \mid t\right)(1-t)^{n}, \quad 0 \leq t<1,
$$

the last expression becomes

$$
L_{n}^{(0)}(t)=\int_{t}^{2 t} n L_{n-1}^{(0)}(\tau) d \tau+\frac{1}{2} \int_{2 t}^{1} n L_{n-1}^{(0)}(\tau) d \tau .
$$

Note that this time change of variables does not help that much, because now we do not only face a recursion in $n$, but also one in $t$. Indeed, if we naturally put

$$
L_{0}^{(0)}(t)=\mathrm{P}\left(K_{0}^{\prime}=0 \mid t\right)(1-t)^{0} \equiv 1, \quad 0 \leq t<1
$$

then for $n=1$ the equations (28) and (27) immediately yield:

$$
L_{1}^{(0)}(t)= \begin{cases}1 / 2=(1-t)-\frac{(1-2 t)}{2}, & 0 \leq t<1 / 2 \\ 1-t, & 1 / 2 \leq t<1\end{cases}
$$

This expression can also be verified directly. Now, to solve $L_{2}^{(0)}(t)$ from $(28)$ we have to distinguish two cases: $0 \leq 2 t<1 / 2$ and $1 / 2 \leq 2 t<1$. Hence, we will have different expressions for $L_{2}^{(0)}(t)$ at $0 \leq t<1 / 4$ and $1 / 4 \leq t<1 / 2$. Proceeding this way, we conclude that it is necessary to consider the intervals $0 \leq t<1 / 2^{n}, 1 / 2^{n} \leq t<1 / 2^{n-1}$, $\ldots, 1 / 2 \leq t<1$ to find $L_{n}^{(0)}(t)$. So, as before, the calculations have to be done 'backwards' (cf. Remark 6.3), because we need to know $L_{n-1}^{(0)}(\tau)$ at $\tau \in[t, 1)$ in order to find $L_{n}^{(0)}(t)$.

Solving the recursion (28) one can see that the function $L_{n}^{(0)}(t)$ has the form

$$
L_{n}^{(0)}(t)=\sum_{i=0}^{n}(-1)^{i} k_{n, i}\left(1-2^{i} t\right)^{n} \mathbf{1}_{\left[0,1 / 2^{i}\right)}(t), \quad 0 \leq t<1,
$$


and it only remains to find the coefficients $k_{n, i}, n=0,1, \ldots ; i=0,1, \ldots, n$, which turn out to satisfy the following recursion:

$$
\begin{aligned}
k_{n, 0} & =1, \quad n=0,1, \ldots \\
k_{n, i} & =\sum_{l=i-1}^{n-1} k_{l, i-1}\left(\frac{1}{2^{i}}\right)^{n-l}, \quad n=1,2, \ldots ; \quad i=1,2, \ldots, n .
\end{aligned}
$$

Thus, those coefficients are just geometric sums:

$$
\begin{aligned}
& k_{n, 0}=1, \quad n \geq 0 \\
& k_{n, 1}=1-1 / 2^{n}, \quad n \geq 1 \\
& k_{n, 2}=1 / 3-1 / 2^{n}+2 / 3 \cdot 1 / 4^{n}, \quad n \geq 2,
\end{aligned}
$$

and so on. Note that we have seen the same coefficients for the conditional mean and variance of the travel time and the number of turns (see Theorems 5.2, 6.1, 9.2 and 9.3).

Now we can apply similar methods to calculate the conditional probabilities $\operatorname{Pr}\left[K_{n}^{\prime}=\right.$ $k \mid t], k=1,2, \ldots, n$, conditioned on the empty space $t$. Since a turn necessarily provides a step, which is greater than the size of the empty space, we may conclude from Lemma 9.1 that the number of turns can only achieve $k$ if $t<1 / 2^{k}$ :

$$
\operatorname{Pr}\left[K_{n}^{\prime}=k \mid t\right]=0, \quad t \geq 1 / 2^{k}
$$

Thus, denoting

$$
L_{n}^{(k)}(t)=\operatorname{Pr}\left[K_{n}^{\prime}=k \mid t\right](1-t)^{n}, \quad 0 \leq t<1, \quad 1 \leq k \leq n,
$$

we obtain the following recursion:

$$
L_{n}^{(k)}(t)=\int_{0}^{t} n L_{n-1}^{(k)}(t+y) d y+\int_{t}^{1 / 2^{k}} n L_{n-1}^{(k)}(2 y) d y+\int_{t}^{1 / 2^{k}} n L_{n-1}^{(k-1)}(2 y) d y,
$$

which can be rewritten as

$$
L_{n}^{(k)}(t)=\int_{t}^{2 t} n L_{n-1}^{(k)}(\tau) d \tau+\frac{1}{2} \int_{2 t}^{1 / 2^{k}} n L_{n-1}^{(k)}(\tau) d \tau+\frac{1}{2} \int_{2 t}^{1 / 2^{k-1}} n L_{n-1}^{(k-1)}(\tau) d \tau .
$$

This recursion can be solved subsequently for $t$ in $\left[1 / 2^{k+1}, 1 / 2^{k}\right),\left[1 / 2^{k+2}, 1 / 2^{k+1}\right), \ldots$, $\left[0,1 / 2^{n}\right)$. The results are presented in the following theorem.

Theorem 10.1 For all $0 \leq k \leq n$ we have:

$$
\begin{aligned}
\operatorname{Pr}\left[K_{n}^{\prime}=k \mid t\right] & =\frac{1}{(1-t)^{n}} \sum_{i=k}^{n}(-1)^{i+k}\left(\begin{array}{l}
i \\
k
\end{array}\right) k_{n, i}\left(1-2^{i} t\right)^{n} \mathbf{1}_{\left[0,1 / 2^{i}\right)}(t), \\
\operatorname{Pr}\left[K_{n}^{\prime}=k\right] & =\sum_{i=k}^{n}(-1)^{i+k}\left(\begin{array}{l}
i \\
k
\end{array}\right) k_{n, i} .
\end{aligned}
$$


According to (25) the conditional distribution of $K_{n}$ for $t>0$ is the same as the one of $K_{n}^{\prime}$, and for $t=0$ it can be found from:

$$
\begin{aligned}
\operatorname{Pr}\left[K_{n}=k\right] & =2 \sum_{l=0}^{k}(-1)^{l} \operatorname{Pr}\left[K_{n}^{\prime}=k-l\right] \\
& =2 \sum_{l=0}^{k} \sum_{i=l}^{n}(-1)^{i+k}\left(\begin{array}{l}
i \\
l
\end{array}\right) k_{n, i}, \quad 0 \leq k \leq n,
\end{aligned}
$$

which is another form of formula (22) from Theorem 8.5.

In Fig. 11 we show the conditional probability $\operatorname{Pr}\left[K_{n}=0 \mid t\right]$ of no turns as a function of the empty space $t$ for $n=10$ (observe the discontinuity at $t=0$ ). We see that it rapidly goes to 1 as $t$ increases. Hence, the picker only oscillates near his starting position. Once he has picked a few items, it becomes very unlikely that he will turn.

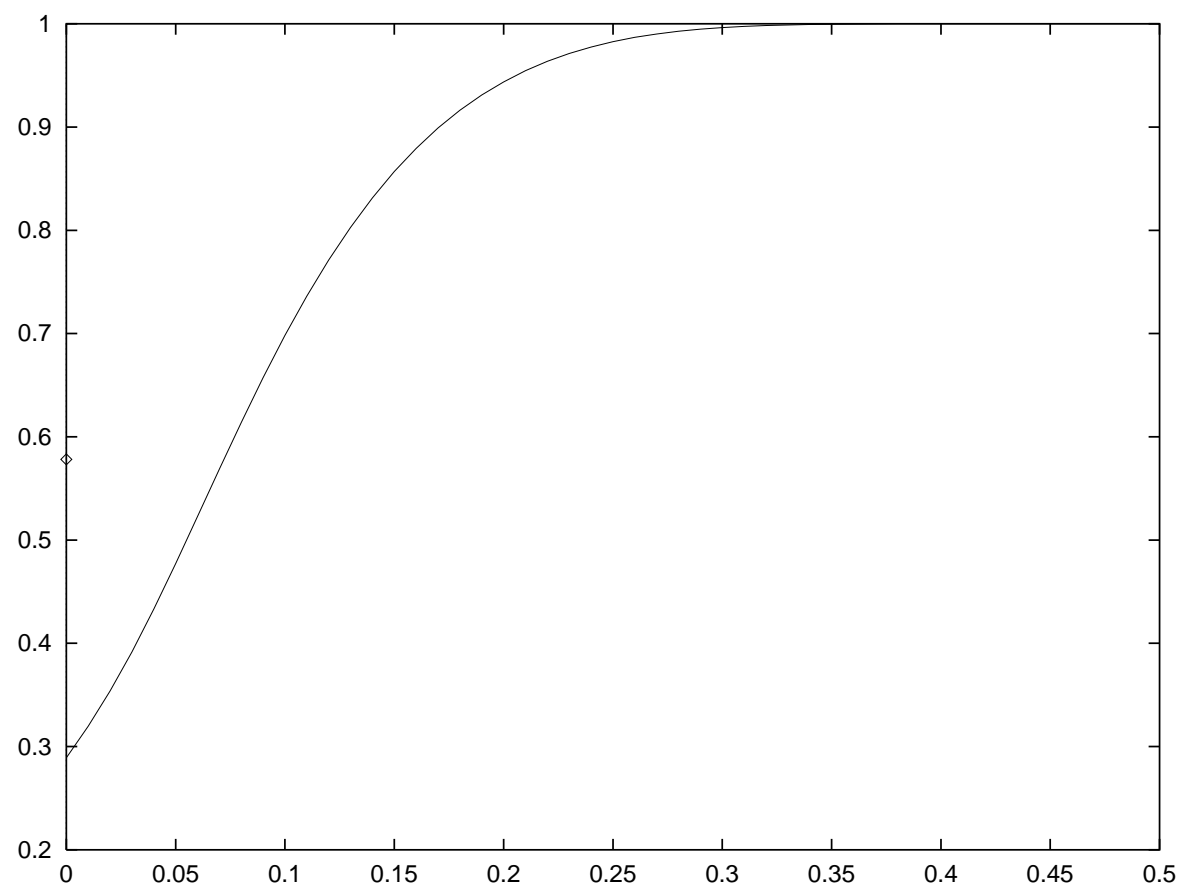

Figure 11: The conditional probability $\operatorname{Pr}\left[K_{n}=0 \mid t\right]$ of no turns as a function of the empty space $t$ for $n=10$.

Letting $n \rightarrow \infty$ in (30) and denoting

$$
k_{\infty, i}=\lim _{n \rightarrow \infty} k_{n, i}=\prod_{j=2}^{i} \frac{1}{2^{j}-1}, \quad i=1,2, \ldots,
$$

we obtain after some simplifications the following elegant expression for the limiting distribution. 
Theorem 10.2 The limiting distribution of $K_{n}$ as $n \rightarrow \infty$ is given by:

$$
\operatorname{Pr}\left[K_{\infty}=k\right]=2 \cdot(-1)^{k} \sum_{i=k+2}^{\infty}(-1)^{i} k_{\infty, i} \sum_{l=0}^{k}\left(\begin{array}{l}
i \\
l
\end{array}\right)
$$

In particular, (31) gives (cf. (23)):

$$
\begin{aligned}
& \operatorname{Pr}\left[K_{\infty}=0\right]=2 \cdot\left\{\frac{1}{3}-\frac{1}{3 \cdot 7}+\frac{1}{3 \cdot 7 \cdot 15}-\ldots\right\} \\
& \operatorname{Pr}\left[K_{\infty}=1\right]=2 \cdot\left\{\frac{1}{3 \cdot 7}(1+3)-\frac{1}{3 \cdot 7 \cdot 15}(1+4)+\frac{1}{3 \cdot 7 \cdot 15 \cdot 31}(1+5) \ldots\right\} \\
& \operatorname{Pr}\left[K_{\infty}=2\right]=2 \cdot\left\{\frac{1}{3 \cdot 7 \cdot 15}(1+4+6)-\frac{1}{3 \cdot 7 \cdot 15 \cdot 31}(1+5+10)+\ldots\right\} .
\end{aligned}
$$

Remark 10.3 Formulas (23) and (32) are of cause the same, which can be proved by combinatorical arguments. Let us show, for example, that the formulas for $\operatorname{Pr}\left[K_{\infty}=0\right]$ from (23) and (32) indeed coincide. In other words, we are going to prove the equality

$$
\prod_{i=1}^{\infty}\left(1-\frac{1}{2^{i}}\right)=\frac{1}{3}-\frac{1}{3 \cdot 7}+\frac{1}{3 \cdot 7 \cdot 15}-\cdots
$$

To do that, we open braces in the left-hand side and we arrange the terms as follows:

$$
\prod_{i=1}^{\infty}\left(1-\frac{1}{2^{i}}\right)=1-\sum_{i=1}^{\infty} \frac{1}{2^{i}}+\sum_{i=1}^{\infty} \sum_{j=i+1}^{\infty} \frac{1}{2^{i}} \cdot \frac{1}{2^{j}}-\sum_{i=1}^{\infty} \sum_{j=i+1}^{\infty} \sum_{k=j+1}^{\infty} \frac{1}{2^{i}} \cdot \frac{1}{2^{j}} \cdot \frac{1}{2^{k}}+\cdots .
$$

Further, note that

$$
\begin{aligned}
\sum_{i=1}^{\infty} \sum_{j=i+1}^{\infty} \frac{1}{2^{i}} \cdot \frac{1}{2^{j}} & =\frac{1}{4}\left(\frac{1}{2}+\frac{1}{4}+\cdots\right)+\frac{1}{16}\left(\frac{1}{2}+\frac{1}{4}+\cdots\right)+\cdots \\
& =\left(\frac{1}{2}+\frac{1}{4}+\cdots\right)\left(\frac{1}{4}+\frac{1}{16}+\cdots\right)=\frac{1}{3}
\end{aligned}
$$

Similarly,

$$
\sum_{i=1}^{\infty} \sum_{j=i+1}^{\infty} \sum_{k=j+1}^{\infty} \frac{1}{2^{i}} \cdot \frac{1}{2^{j}} \cdot \frac{1}{2^{k}}=\left(\frac{1}{2}+\frac{1}{4}+\cdots\right)\left(\frac{1}{4}+\frac{1}{16}+\cdots\right)\left(\frac{1}{8}+\frac{1}{64}+\cdots\right)=\frac{1}{3 \cdot 7},
$$

and so on. Thus, the left-hand side of (33) becomes

$$
1-1+\frac{1}{3}-\frac{1}{3 \cdot 7}+\frac{1}{3 \cdot 7 \cdot 15}-\cdots
$$

which is the required result. 


\section{Discussion}

One may distinguish two main directions for studying the performance of carousel systems. The first one concerns the analysis of the optimal order picking strategy. However, a detailed analysis of probabilistic characteristics of the response time is quite complicated (cf. Rouwenhorst et al. [5]).

The other main direction is developing and studying simple heuristics for order picking in carousel systems. In practice they can be very useful, because they provide reasonable control without much (computational) effort. Probabilistic properties of such heuristics sometimes can be obtained analytically. So, in real life one may prefer simple heuristics because (a) they don't require much effort, and (b) their properties are well-understood.

The present paper can be classified in the second direction. We studied in detail the NI heuristic. We provided a tight upper bound for the travel time. We used probabilistic arguments to find the mean travel time and the distribution for the number of turns. Moreover, in Section 5 we developed a procedure to obtain the conditional mean and variance of the travel time and also the conditional distribution for the number of turns given that there is a certain empty space at one side of the picker's position. Also, we gave a quite accurate two-moment approximation for the distribution function of the travel time under the NI heuristic.

Acknowledgment. The authors thank Jacques Resing for some very useful suggestions and stimulating discussions.

\section{References}

[1] J.J. Bartoldi, III And L.K. Platzman. Retrieval strategies for a carousel conveyor. IIE Transactions, 18(2): 166-173, 1986.

[2] J.P. VAN DEN BERG. Planning and Control of Warehousing Systems, Ph.D. thesis, University of Twente, Faculty of Mechanical Engineering, 1996.

[3] R. Pyke. Spacings. J. Roy. Statist. Soc., B. 27: 395-436, 1965.

[4] R. Pyke. Spacings revisited. In 6th Berkeley Symp., Math. Statist. Prob., 1: 417-427, 1972.

[5] B. Rouwenhorst, J.P. van den Berg, G.J. van Houtum, and W.H.M. Zijm. Performance analysis of a carousel system. In Progress in Material Handling Research: 1996, The Material Handling Industry of America, Charlotte, NC, 1996, pp. 495-511.

[6] H.I. Stern. Parts location and optimal picking rules for a carousel conveyor automatic storage and retrieval system. In 7th International Conference on Automation in Warehousing, pp. 185-193, 1986. 